\title{
Validity of Laws of Electrodynamics
}

$\mathrm{N}$ the course of an investigation carried out for an electrical engineering company, I found that some generally accepted laws of electrodynamics were incorrect, and that serious mistakes were therefore being made by electrical engineers. As the laws here

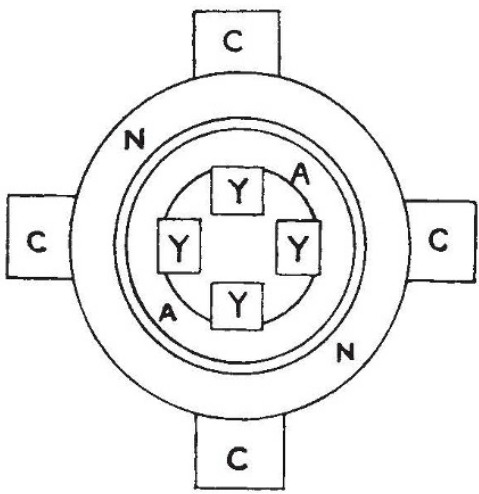

Fig. 1.

referred to have been taught by such high authorities as Maxwell and Jeans, they are naturally being repeated in most of the text-books that are now used at our universities and technical colleges : in fact, one of these laws (which has already caused patents to be taken out for circuit breakers that will work in a very different way from what was expected, and for electric motors that will not work at all) is taught in all text-books that deal with the subject, including those used on the Continent and in America.

The law to which I have just referred is sometimes ascribed to Laplace, sometimes to Biot and Savart, sometimes to Ampère, and sometimes to Maxwell; but all teachers appear to regard that law as infallible. It may be stated as follows, though it is more frequently stated in a less complete form.

When a straight conductor element of length $d x$ carries a current $i$ in the $O X$ direction of rectangular co-ordinates (right-handed) and lies in a magnetic field where the flux density has a component $b$ in the $O Y$ direction, it is acted upon by a force $i . b . d x$ in the $O Z$ direction.

The units here referred to are, of course, those of the c.G.s. system usually employed in text-books of electromagnetism. The current is accordingly in dekamperes and the force in dynes. In view of the doubt with regard to the name of the investigator by whom this law was first enunciated, I propose calling it 'the $i . b$ law'. This name seems appropriate, since the law says that $i . b$ is the force intensity (dynes per centimetre length of conductor) at the point in question.

But when the $i . b$ law is applied to the case shown in Fig. 1, which is an end-view of a direct-current slip-ring dynamo designed by Dr. A. E. Clayton, it tells us that the machine will work, though Dr. Clayton found that it would not. From his description of the machine, in the Electrician of July 2, 1915, it appears that he may not have designed it with the idea that it would work, but with the idea that its failure would disprove Faraday's law of electromagnetic induction. He evidently overlooked the fact that its failure has disproved the $i . b$ law; for he continued to teach this law, which is repeated in his latest books on dynamo design. But the following considerations will make it clear that the $i . b$ law has been conclusively disproved by the failure of this machine.

In Fig. 1, $C, C, C, C$ are the ends of the straight iron cores upon which the field coils are wound, $N$ is the ring-shaped north pole of the magnet, $A$ is the ring-shaped core of the armature, and $Y, Y, Y, Y$ are the ends of the straight iron yokes that connect this armature to a similar one at the south pole end and rotate with these armatures. All the rings here referred to were of rectangular cross-section, and the armatures were ring-wound on the parts between the yokes.

The machine should evidently work as a motor, according to the $i . b$ law, when the windings are suitably fed with direct current through the sliprings ; for the conductors that are in the main flux (between the armature cores and the magnet poles) should be subjected to a force much greater than any opposing force acting on conductors in the leakage flux, owing to the great permeance of the magnetic path in the iron yokes. Actually, however, the machine would not work; and this will be under-

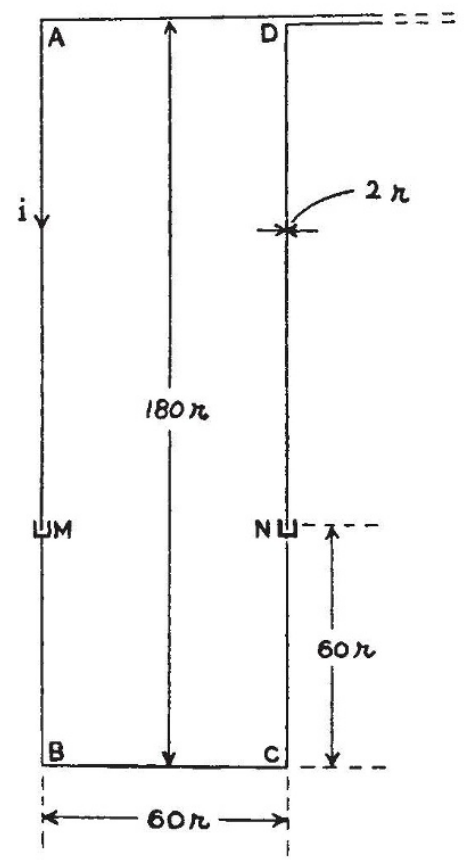

Fig. 2.

stood when attention is directed to the fact that the amount of flux linking the winding would not begin to change when the armature began to rotate, with the consequence that (according to Neumann's law) no back-e.m.f. would be generated. 
Dr. Clayton eventually came to the conclusion that his machine was a failure from every point of view : that is to say, it not only failed to work, but also (in his opinion) failed to give a disproof of Faraday's law. His present view is that Faraday's "lines of magnetic force" were rushing from yoke to yoke when the armature was rotating, cutting the inside conductors on their way, and thereby generating an e.m.f. that counterbalanced the one generated in the outer conductors. Many electrical engineers are unable to see any justification for this view; and Dr. Clayton will certainly find it useless as a defence of Faraday's law against the following argument.

Fig. 2 shows an experimental circuit-breaker arrangement, with mercury cups at $M$ and $N$ (where the arcs would be formed when the actual circuit breaker began to open). When this arrangement is surrounded by air, or by any other medium of unit permeability in the c.G.s. electromagnetic system, the amount of flux $d c$ that is cut during a downward movement of $M B C N$ through the distance $d y$, with the current kept constant, can be calculated by methods explained in text-books of electromagnetism. It will thus be found that $d c$ consists of a part

$$
2 i d y(0 \cdot 054+0 \cdot 161)
$$

that comes from $M A D N$ and is cut by $B C$, and a part

$$
2 i d y(0 \cdot 054+0 \cdot 030)
$$

that comes from $M B C N$ and is pulled across $A D$, so that

$$
d c=0 \cdot 6 i d y \text {. }
$$

According to Maxwell (see Art. 541 of his "Treatise on Electricity and Magnetism"), the total amount of flux-cutting must be equal to the total increase in the flux that links the electric circuit. But when we try to verify this in the present case, by making a direct calculation of the extra flux linkage $d k$ that is due to the downward movement of $M B C N$ through the distance $d y$, we get

$$
d k=17 \cdot 5 i d y \text {. }
$$

The great difference between $d c$ and $d k$ is accounted for by the fact that the stretching of $A B$ and $D C$ through the distance $d y$ causes additional flux to be sent through the area $A B C D$ by the added lengths $d y$ at $M$ and $N$.

If $M B C N$ is moving downwards with a velocity of $v$ centimetres per second, and if the movement through the distance $d y$ takes place in the time $d t$, we have $d y / d t=v, d c / d t=0.6 i v$, and $d k / d t=17.5 i v$. The induced E.M.F. will therefore be $0.6 i v$ or $17.5 i v$, according to whether Faraday's law or Neumann's law is correct. To settle this question, I attached $M B C N$ to one end of a strip of wood that was arranged to turn about a knife-edge in the manner of the beam of a balance. This enabled me to measure the electromagnetic force $F$ exerted on $M B C N$ when various currents (up to 1,300 amperes) were flowing in the circuit. The strip of wood was fastened to the middle of $B C$, and was at right angles to the plane of $A B C D$ when in the position of equilibrium.

As will be seen from Maxwell's Art. 583 or my article in World Power of March 1932, the energy equation is either

or

$$
\begin{aligned}
& F d y=\frac{1}{2} i d c, \\
& F d y=\frac{1}{2} i d k, .
\end{aligned}
$$

according to whether Faraday's law or Neumann's law is correct; and a measurement of $F$ with any given value of $i$ will show whether (1) or (2) is the correct equation. In each of the numerous cases that were tried, with various ratios of $B M$ to $B C$ and of $B M$ to $B A$, the measured force was in close agreement with equation (2), though it was frequently (as in the case of Fig. 2) between 20 and 30 times as great as the force indicated by equation (1).

It may therefore be concluded, not merely that there is sometimes a great difference between the $d c$ and $d k$ that Maxwell thought to be identical, but also that Neumann's law is right and Faraday's is wrong.

$$
\text { W. F. Dunton. }
$$

DURING recent years the points raised by Mr. Dunton in the above note have been discussed at considerable length in electrical journals. Indeed, controversy over the laws of electromagnetic induction has flared up periodically during the last half century. About forty years ago Carl Hering devised a number of ingenious experiments to show that 'flux cutting' was the essential factor in the generation of electromotive force. Some twenty years later, Blondel described in the Electrician an experiment in which the total flux linkage-as defined by the product of the flux and the number of turns-was reduced from a large value to zero without E.M.F. being induced in the circuit, his method being to unwrap a coil which had been wound upon a cylindrical magnet. A few years prior to this, I had carried out a number of experiments in an attempt to establish discrepancies between the 'flux cutting' and the 'rate of change of flux' theories, and in the correspondence consequent upon the publication of Blondel's work, I described one of the models I had made for that purpose.

This model is the one described above by $\mathrm{Mr}$. Dunton; before it was built it was anticipated that no E.M.F. would be generated in its winding, and its behaviour on test fulfilled those expectations. How. ever, I finally concluded that the action of the model was in line with that of the slotted armature gene. rators now universally used, and the result of my investigations was to lead me to conclude that the total amount of 'flux cutting' is always equal to the change in the flux linkages. In short, my con. clusions were in agreement with Art. 541 of Max. well's classic book.

The behaviour of the model as a motor was never considered, but it follows that if its behaviour as a generator is in line with the slotted armature machine, such will also be the case for motor action. The action of motors with slotted armatures is well known, and it is also well known that the torque developed between stator and rotor is altogether greater than would correspond to the product of the current in the conductors and the flux density in the slot. In my opinion the model gave no evidence regarding the laws of electromagnetic induction and electrodynamics other than that long since available with slotted armature machines.

For the past few years, Mr. Dunton has, in a most energetic and far-reaching manner, challenged the validity of some of the commonly accepted laws. His work arises out of calculations connected with expanding circuits-such calculations being of particular importance in the design of circuit-breakers. One such example is given in Fig. 2 of Mr. Dunton's communication. Now the flux linked with a circuit may be changed in various ways, of which one $(a)$ is by changing the current carried by the circuit, and 